\title{
Osservazioni sulla rima finale del «Desconhort» di Ramon Llull
}

\author{
SIMONE SARI
}

Nel 1966 il professor Mario Ruffini pubblicò in Estudios Lulianos ${ }^{1}$ un interessante studio del Plant de nostra Dona di Ramon Llull. Il progetto iniziale, molto più ampio, prevedeva di analizzare integralmente l'uso delle rime nelle poesie del Dottore Illuminato, ma: «La vastità dell'indagine [...] mi ha indotto a restringere l'esame al solo Plant, limitandolo allo studio dei mezzi dei quali si è avvalso il Lullo per ottenere la rima finale. $»^{2}$ Ruffini scelse il Plant poiché prima poesia in alessandrini del beato. In effetti, nell'edizione da lui adottata ${ }^{3}$, l'opera è datata nel 1275. L'attuale stato delle ricerche ${ }^{4}$ porta invece a collocarlo nel 1290-93, periodo in cui Llull produsse le sue più importanti opere mariane: il Llibre de Sancta Maria e le Hores de nostra Dona. Il primato spetta perciò alle Regles introductòries a la pratica de l'Art dimostrativa che, secondo il catalogo stabilito da Bonner, risalirebbero al 1283-55. Ripromettendomi di esaminare le rime finali anche di quest'ultima poesia e di completare il panorama dei poemi in alessandrini della letteratura catalana medievale secondo il repertorio di Parramon i Blasco ${ }^{6}$, con l'analisi del Sermo di Muntaner ${ }^{7}$, in questo studio mi occuperò in particolare del capolavoro poetico di Llull: $L o$ desconhort.

Scritto probabilmente a Roma nel 1295, quindi contemporaneamente all'Arbre de Sciència, con il prologo del quale presenta molte affinità, il

${ }^{1}$ M. Ruffini, Osservazioni sulla rima finale del «Plant de Nostra Dona Santa Maria», in EL 10 (1966) pp. 129-140; 11 (1967) pp. 21-30.

${ }^{2}$ Idem p. 129.

${ }^{3}$ R. Llull, Poesies, a cura di R. d'Alòs-Moner, Els Nostres Clàssics, Barcino, Barcelona 1928.

4 A. Bonner, Obres selectes de Ramon Llull (1232-1316), 2 vols, Moll, Palma de Mallorca, 1989 pp. 553-554. Il catalogo è continuamente aggiornato nel database Ramon Llull (DBLlull): http://orbita.bib.ub.es/llull.

${ }^{5}$ Idem p. 549.

${ }^{6}$ J. Parramon i Blasco, Repertori mètric de la poesia catalana medieval, Curial Edicions Catalanes, Publicacions de l'Abadia de Montserrat, Barcelona 1992.

7 Analisi inclusa nella mia tesi di dottorato sul Plant de Nostra Dona, in corso presso la Scuola di Dottorato Europea in Filologia Romanza presso l'Università degli Studi di Siena. 
Desconhort è il frutto della disperazione del beato per gli insuccessi e i continui rifiuti che la sua opera aveva ottenuto. La forma adottata per rappresentare il proprio sconforto è quella del dibattito tra l'autore e un eremita, introdotto da cinque strofe nelle quali il narratore-Ramon introduce se stesso e le cause del proprio malessere. Il poema si avvicinerebbe così alla tenso o al partimen provenzale, anche se non ne condivide l'assetto metrico su base strofica affine alla canzone cortese. Infatti, Llull avverte che il Desconhort: «canta's en lo so de Berart» ${ }^{8}$, una canzone di gesta francese, identificata con la Chanson des Saisnes o Chanson des Saxon di Jean Bodel ${ }^{9}$. La tecnica del contrafactum era una caratteristica del sirventese e Llull ne fece un ampio utilizzo ${ }^{10}$, sfruttando: «l'illusione che per chiunque - per chi trasmette, per suo specifico ufficio, poesia, ma anche per chi l'ascolta e la memorizza- appropriarsi del canto significhi pure appropriarsi di tutta la realtà che il canto stesso sembra sottintendere.» ${ }^{11}$ Questa appropriazione non ha, naturalmente, lo stesso obiettivo che si proponevano i cantori della fin'amor. Per Llull lo scopo era principalmente l'estesa circolazione delle proprie opere con tutti i mezzi possibili $\mathrm{e}$, come vedremo, il beato attingerà abbondantemente nella tradizione letteraria del suo tempo per raggiungere i propri scopi. Lo schema metrico del Desconhort, identico a quello del Plant, di pochi anni precedente, è dunque basato su quello dell'epica francese: ogni strofa monorima è composta di 12 versi di 12 sillabe, nel Plant sono 32, nel Desconhort 69. L'edizione adottata è quella di Josep Batalla ${ }^{12}$, che modernizza l'ortografia e fa alcune correzioni accettabili a quella di Amedée Pagès ${ }^{13}$, quest'ultima tradotta in italiano dal prof. Ruffini ${ }^{14}$.

A differenza del Plant, il Desconhort presenta una prevalenza di rime verbali, dato che concorda con i risultati dell'analisi compiuta da Jordi Rubió i Balaguer sulle rime finali delle prose rimate di Llull ${ }^{15}$. Delle 828 parole in rima (o rimanti ${ }^{16}$ ) 433 sono verbali, pari al 52,3\%, mentre le nominali sono 395, quindi il 47,7\%; nel Plant invece le nominali equivalgono al 55,2\% contro il restante $44,8 \%$ di rime verbali. Le rime sono 20 , contro le 17 del Plant quindi, in proporzione, c'é minor varietà. Le rime esclusive del Desconhort sono -ança, às, -è, -er, -ets, -it e -ort; quelle del Plant -ada, -el, -í, e -ots. Le rime più usate nel

${ }^{8}$ R. Llull, Lo desconhort, Cant de Ramon, a cura di J. Batalla, Obrador Edèndum, Barcelona 2004, p. 129.

9 Ibidem.

${ }^{10}$ I Cent noms de Déu sono cantati sulle melodie dei Salmi, mentre le Hores de nostra Dona su quelle degl'Inni, purtroppo non abbiamo attestazioni riguardo al Plant.

${ }_{11}$ M. L. Meneghetti, Il pubblico dei trovatori, Einaudi, Torino 1992, p. 69.

12 R. Llull, Lo desconhort, Cant de Ramon, cit.

13 A. Pagès, Le «Desconort» ou le «Découragement» de Ramon Llull, in Annales du Midi, 50 (1938) pp. 113-156, 225-267.

${ }^{14}$ R. Llull, Lo sconforto, a cura di M. Ruffini, Il Melograno, Fussi Sansoni, Firenze 1953.

15 J. Rubió i Balaguer, Sobre la poesia rimada de Ramon Llull, in Estudios dedicados a Menéndez Pidal V, Madrid 1954, pp. 307-318 [anche in id., Ramon Llull i el lu lisme, Publicacions de l'Abadia de Montserrat 1985, pp. 234-247].

${ }_{16}$ Per l'uso di questo termine cfr. R. Antonelli, Equivocatio e repetitio nella lirica trobadorica, in Id., Seminario romanzo, Bulzoni, Roma 1979 (in particolare pp. 115-116). 
Desconhort sono -at (15 strofe), -ar (11), -ent (11) e -or (7), nel Plant -ent (5), -at (4), -ats (3), e -ia (3). In entrambi i poemi le rime esclusivamente nominali sono due: nel Desconhort -ança e -ors (-os) e nel Plant -el e -ors (-os). Questo dato è particolarmente interessante perché -ança e -el sono rime esclusive dei due poemi, mentre la rima comune -ors (-os) è nominale in entrambi. Mancano nel Plant rime esclusivamente verbali, che invece sono rappresentate dalla strofa in -ets nel Desconhort. Inoltre si nota la stessa proporzione percentuale della rima in -ut in entrambi i poemi. Questi dati si possono riassumere nella tabella che segue, nella quale ho confrontato i risultati della mia indagine con quelli riportati nell'articolo di Ruffini ${ }^{17}$.

DESCONHORT

\begin{tabular}{|l|c|c|}
\hline \multicolumn{1}{|c|}{ Rima } & Nominale & Verbale \\
\hline- & - & - \\
-al & 9 & 3 \\
-ança & 12 & - \\
-an, -ant & 11 & 13 \\
-ar & 10 & 122 \\
-às & 2 & 10 \\
-at & 71 & 109 \\
-ats & 7 & 17 \\
- & - & - \\
-è & 11 & 13 \\
-ent & 118 & 14 \\
-er & 14 & 22 \\
-es & 3 & 9 \\
-ets & - & 12 \\
- & - & - \\
-ia & 8 & 16 \\
-ir & 3 & 21 \\
-it & 4 & 20 \\
-ó & 28 & 8 \\
-or & 47 & 1 \\
-ors (-os) & 24 & - \\
-ort & 9 & 3 \\
- & - & - \\
-ut & 4 & 20 \\
Totali & 395 & 433 \\
\hline
\end{tabular}

Plant de LA Verge

\begin{tabular}{|l|c|c|}
\hline \multicolumn{1}{|c|}{ Rima } & Nominale & Verbale \\
\hline -ada & 3 & 9 \\
-al & 11 & 1 \\
- & - & - \\
-an, -ant & 9 & 3 \\
-ar & 2 & 22 \\
- & - & - \\
-at & 15 & 33 \\
-ats & 12 & 24 \\
-el & 12 & - \\
- & - & - \\
-ent & 56 & 4 \\
- & - & - \\
-es & 5 & 7 \\
- & - & - \\
-í & 9 & 15 \\
-ia & 8 & 28 \\
-ir & 1 & 11 \\
- & - & - \\
-ó & 20 & 4 \\
-or & 11 & 1 \\
-ors (-os) & 24 & - \\
- & - & - \\
-ots & 11 & 1 \\
-ut & 2 & 10 \\
Totali & 211 & 173 \\
\hline
\end{tabular}

17 M. Ruffini, Osservazioni, cit. p. 131. 
Per quanto riguarda l'uso dei rimanti troviamo gli stessi artifici usati nel Plant, come l'uso delle stesse parole in rima nella medesima strofa (20 nominali e 20 verbali):

Nominali: desconhort 1,1 e 7; natural 8,3 e 8; bé 9,2 e 11; pecat 12,6 e 10; discreció 16,1 e 11; raó 16,2 e 8; bo 20,5 e 12; fe 25,1 e 7; argument 28,2 e 7; opinió 37,2 e 6; caritats 42,8 e 11; predestinat 45,2 e 12; llibertat 45,3, 5 e 11; fiança 46,7 e 11; via 48,7 e 9; pecador 49,2 e 7; uniment 56,4 e 10; tractament 56,6 e 12 ; veritat 57,1 e 8 ; crestiantat 63,3 e 9.

VERBALI: venia 5,2 e 7; havia 5,3 e 6; ajut 6,6 e 12; val 8,5 e 7; cové 9,1 e 4; só 16,4 e 9; acabar 17,2 e 12; conegut 21,1 e 12; far 23,4 e 12; ausir 35,1 e 10; dir 35,3 e 11; creat 38,4 e 11; consolar 39,1 e 5; sia 48,6 e 11; haver 52,2, 3 e 10 ; treballats 54,6 e 8; ordenat 55,7 e 12; haver 58,10 e 12; havets 60,3, 10 e 11; escomès 62,3 e 9.

La caratteristica riscontrata da Ruffini nel Plant, ossia di non trovare mai la stessa parola ripetuta nel verso seguente, è elusa in un solo caso verbale: haver 52,2 e 3. Inoltre il Desconhort presenta tre casi, di cui uno nominale e due verbali, nei quali la parola-rima è ripetuta tre volte: llibertat 45,3, 5 e 11; haver 52,2, 3 e 10; havets 58,10 e 12; fenomeno che nel Plant non si riscontra.

Gli esempi di rime omofone o equivoche ${ }^{18}$, più difficili da conseguire, sono di tre tipi:

a) tra verbale e nominale:

port (nominale) 1,6 e port (verbale) 10; pecat 12,1 (verb.) e pecat 6 (nom.); creat 26,1 (verb.) e creat 26,8 (nom.);

b) tra due nominali:

semblant 11,1 e semblant 11,12 ;

c) tra due verbali:

ve (venir) 9,3 e ve (veure) 9,5; ripetuto ai versi 25,5 e 6 .

L'uso di corradicali in rima o di rime derivative è più diffuso che nel Plant: desconhort 1,1 e conhort 1,2; conhort 1,2 e confort 1,12 che mostra l'esito popolare e colto della stessa parola; deport 1,8 aport 1,9 e port 1,10 ; deshonor 4,2 e honor 4,10; conegut 21,1 e desconegut 21,8; cové 25,2 e descové 25,12; creat 26,8 e increat 26,9; honor 49,4 e deshonor 49,5; pacient 50,1 e impacient 50,10; obtener 58,2, tener 58,7, mantener 58,9 e cartener 58,11; aportar 61,2 e portar 61,3: estés 62,2 e estegués 62,10; captener 64,2, retener 64,7 e mantener 64,8; dit 69,2 e contradit 69,11.

${ }_{18}$ Per le rime equivoche sono fondamentali gli studi di Roberto Antonelli, Equivocatio e repetitio, cit. e id., Rima equivoca e tradizione rimica nella poesia di Giacomo da Lentini. I. Le canzoni, in Bollettino del Centro di Studi Filologici e Linguistici Siciliani, 13 (1977) pp. 20-106. 
La rima derivativa è inoltre usata per ottenere dei concetti contrari in rima, anche se questo artificio viene usato solo in otto casi su ventidue, come si nota nei seguenti esempi:

desconhort 1,1 e conhort 1,2; desconhort 1,7 e confort 1,12 ; deshonor 4,2 e honor 4,10; perdut 6,1 e vençut 6,10; espirital 8,2 e terrenal 8,12; virtuós 10,10 e viciós 10,11; malestant 11,4 e benestant 11,5; Preicadors 14,6 e Menors 14,7 che si ripete nella strofa 32, 1 e 2; llausar 19,5 e menysprear 19,6; conegut 21,1 e desconegut 21,8; oblidàs 22,7 e membràs 22,10; cové 25,2 e descové 25,12; creat 26,8 e increat 26,9; menor 32,2 e major 32,8; fadigat 33,5 e reposat 33,11 ; acabar 36,6 e començar 36,8; salvats 42,9 e damnats 42,10; alegrança 44,6 e tristança 44,10; honor 49,4 e deshonor 49,5; crestianitat 63,3 e 9 e sarrainitat 62,8; dit 69,2 e contradit 69,11.

$\mathrm{Al}$ contrario i sinonimi in rima non sono molti:

ociós 14,1 e pererós 14,11; ausit 29,3 e sentit 29,5; consolar 39,1 e 5 e confortar 39,12 ; justament 40,1 e dretament 40, 12; consolat 51,1 e conhortat 51,12.

Come si potrà vedere nel rimario, che conclude il presente articolo, alcuni degli 828 rimanti del Desconhort si ripetono in più strofe, di questi 130 sono nominali e 128 verbali. I primi corrispondono a 76 aggettivi, pronomi e sostantivi e i secondi a 70 forme verbali. Sottraendo al totale delle rime nominali le parole ripetute si ottengono 265 rime e con lo stesso procedimento si riscontrano 305 rime verbali, naturalmente tenendo conto delle rime equivoche che hanno valore a sé. Il rimario del Desconhort è quindi composto da 570 parole pari al 69\% del totale, mentre nel Plant la stessa analisi aveva condotto all' $89 \%$ del totale. Dal confronto dei due rimari si evidenzia inoltre che Llull riutilizza nel Desconhort 92 rimanti già del Plant. Sono escluse sia le cinque strofe con rime non presenti nel Desconhort: 7 (-ada), 24 (-el), 19 e 26 (-1́), 25 (-ots); sia sei con rima comune che presentano il prestito di alcune parole che nel Plant non rimano nella stessa strofa: 17 e 27 (-at), 12, 14 e 28 (-ats), 29 (-ent). Questi dati sono esemplificati nella seguente tabella. Sono da notare in particolare la str. 2 del Plant che presenta nove parole comuni, delle quali otto sono usate per costruire la str. 6 del Desconhort; e la str. 9 del primo che mostra sei parole interamente riprese nella strofa 57 del secondo.

\section{Plant}

str. 1: Maria 1, via 10, sia 11.

\section{Desconhort}

str. 48: Maria 3, sia 6 e 11, via 7 e 9.

str. 2: haut 2 , vertut 3 , volgut 4 , decebut 5 , str. 6: perdut 1 , salut 2 , vertut 4 , abatut 5, haut 7, decebut 8, perdut 6 , salut 8 , abatut 9 , combatut 10 , vençut 10, combatut 11; str. 21 : decebut 2 , volgut 4 ,

vençut 12.

str. 3: fo 4 e 7, so 9 .

str. 4: compliment 3, follament 5 .

str. 5: val 2, cabal 5, natural 7, espirital 10, str. 8: espirital 2, natural 3 e 8, val 5 e 7 , mortal 10, cabal 11. vertut 9 , salut 10 , perdut 11 .

str. 20: so 7, fo 10; str. 37: fo 3, so 11.

str. 7: compliment 1, follament 9 . 
mortal 12.

str. 6: res 3, pogués 4.

str. 62: res 5, pogués 8 .

str. 8: major 2, creador 3, senyor 6, valor 9, str. 4: amor 3, senyor 4, plor 5, valor 12; str. 32: valor 5, major amor 10, plor 12 . 8, creador 12; str. 41: senyor 1, amor 4, plor 8; str. 49:

amor 1 , senyor 3 .

str. 9: veritat 3 , pecat 6 , honrat 8 , amat 9, str. 2 : pecat 2 , pietat 4 , veritat 8 , honrat 11; str. 12: pecat $6 \mathrm{e}$

donat 10 , pietat 12 . 10 , amat 11 ; str. 26 : veritat 2 , honrat 3 ; str. 30 : amat 2 , pecat 8 ; str. 44 : pecat 9 , veritat 12 ; str. 55 : amat 1 , honrat 5 , donat 9 ; str. 57 : veritat 1 e 8, pietat 4, traballat 5, donat 10; str. 68: pietat 4, amat 7 .

str. 10:havia 3, podia 9 e 12, venia 11 . str. 5: venia 2 e 7, havia 3 e 6, podia 8.

str. 11: jaquir 3, servir 4, fallir 5. str. 31: servir 5, fallir 7, jaquir 11.

str. 13: par 1, estar 4, escoltar 8, far 11. str. 17: far 1, estar 7; str. 23: far 4, par 5; str. 34: far 9, estar 10; str. 59: far 4, estar 5, escoltar 6; str. 61: far 8, estar 12; str. 65: estar 7, far 11.

str. 15: pietat 4 , jutjat 8 , amat 11 , treballat 12 . str. 45 : amat 9 , jutjat 10; str. 57: pietat 4, treballat 5; str. 68: pietat 4, treballat 6, amat 7 .

str. 16: turment 2, malament 7.

str. 40: malament 5, turment 9.

str. 18: turment 1 e 9 , honrament 4 , vivent 10, str. 43 : turment 2 , honrament 12; str. 50: turment 4, honrament

gent 12. 12; str. 53: honrament 3, gent 9; str. 56: gent 3, vivent 7.

str. 20: coman 3, afan 9, plorant 11, deman 12. str. 67: afan 1, plorant 5, deman 6, coman 8.

str. 21: sia 9, dia 12 .

str. 48: dia 5, sia 6 e 11.

str. 22: turment 1, falliment 7, llanguiment. 11 . str. 40: turment 9, falliment 10; str. 43: turment 2, llanguiment 4

str. 23: llangors 5 , senyors 7 , pecadors $10 . \quad$ str. 14: pecadors 2 , llangors 4 , senyors 9 .

str. 30: sermó 5, pro 6, raó 7 . str. 16: pro 6, sermó 10; str. 20: pro 6 raó 8.

str. 31: consolar 3, estar 4, consellar 9, esforçar 5, str. 13: membrar 2, esforçar 11; str. 17: estar 7, consellar 10; str.

membrar 6, amar 11, parlar 12. 34: amar 4, consellar 5, estar 10; str. 39: consolar 1 e 5, parlar 4, membrar 6; str. 65: membrar 1, estar 7.

str. 32: pecadors 2, menors 3, llangors 6, senyors 8. str. 14: pecadors 2, llangors 4 , menors 7 , senyors 9 . 
La poesia lulliana rispecchia dunque i canoni della tradizione lirica del suo tempo, sa sfruttarne gli artifici e la tecnica per ottenere gli obiettivi prefissati, cioè la sostituzione della lirica d'amore profana con la nuova poesia d'amore a Dio e alla Vergine, naturalmente rappresentato secondo i canoni dell'Arte.

Ritornando alla nostra indagine, passiamo all'analisi dei fenomeni morfologici utilizzati per ottenere la rima che sono, come nel Plant, l'uso del singolare per il plurale:

descreent 3,2 e 40,2; ajut 6,6 ; decebut 6,7 ; conegut 21,1 ; unit 29,6; errat 30,10 ; preicador 32,1 ; menor 32,2 ; prior 32,3 ; coneixent 36,2 ; vivent 36,10 e 56,7 ; seguent 36,12; visió 37,10; desobeent 40,7; pecador 41,10 e 49,2 e 7; predestinat 44,1 e 45,12; salvat 44,2; llausor 49,10; vestiment 50,8; heretat 51,2; lletrat 55,3 ; prelat 55,10 e 63,4; ordenat 55,12; joglar 61,7; girat 63,6; abraçat 66,2; assemblat 66,9 ; an 67,2 ; departit 69,9 ;

e viceversa:

malvats 42,4; pietats 42,6; caritats 42, 8 e11; honrats 54,2; menats 54,5; desviats 54,9; amats 54,12; perdets 60,12.

Questi esempi si possono ricondurre alla declinazione bicasuale provenzale, per esempio preicador al v. 32,1 e menor v. 32,2 sono entrambi plurali retti, mentre i corrispondenti preicadors v. 14,6 e menors v. 14,7 sono plurali obliqui. Sarebbero quindi grammaticalmente corretti ma, a volte, ritroviamo nello stesso verso parole declinate sia seguendo le regole grammaticali occitane sia quelle catalane, per esempio al v. 3,2: com són paucs crestians e molt li descreent o v. 45,12: ni llibertat costreny prescits ne predestinat. Si nota come l'uso della forma provenzale corrisponda sempre al rimante, sembra quindi usata soprattutto per motivi di rima, anche se troviamo casi nei quali l'uso del plurale retto si allunga in più versi: es. vv. 32, 1-3 N'ermità, si fossen pauc li preicador,/e li clergue seglar e li frare menor,/e encara li monge, tant abat e prior,/.

Altre forme provenzali che troviamo in rima sono:

VERBALI: fo 20,10; 37,1 e 3 per fou; far 17,1; 23,4 e 12; 24,12; 34,9; 59,4; 61,8; 65,11 per fer (ma fer 64,11); ausir 31,3; 35,1 e 10 ausit 29,3 aujats 42,3 per oir, oüt e oüu; aunir 11,6 aunit 29,12 per ultratjar e ultratjat; altri es. llausar 19,5; jaquir 31,11; 35,2.

Nominali: dessé 9,6; 25,4 per de seguida; gabament 47,10 per befa, marriment 47,6; 53,7 per malencolia, jausent 53,4 per joiós (ma joiós 10,2); altri es. austor 49,10 .

Due sono i casi di velarizzazione della laterale, tipica dell'occitano ma non del catalano: exauçament 15,8 (ma exalçament 3,8); autrejat 44,11.

Questo lungo elenco di fenomeni dimostra come Llull sfrutti a pieno le proprie capacità tecniche di poeta più nel Desconhort che nel Plant, quasi come se quest'ultimo fosse una specie di prova generale, applicata a un genere molto 
conosciuto nel campo romanzo ${ }^{19}$, per poi dare il meglio nella sua opera poetica più personale.

$$
* * *
$$

Dall'analisi dei fenomeni lessicali e morfologici passiamo a quella della sintassi. Il mezzo sintattico più utilizzato nei due poemi è l'uso delle proposizioni relative, che, come si vede negli esempi seguenti, può occupare il secondo emistichio del verso o si può restringere a un trisillabo ovvero allungarsi al verso intero. Riportiamo solo le relative che si trovano o influiscono sulla rima finale.

I. ESEMPI DI PROPOSIZIONI RELATIVE ESASILLABICHE SECONDO EMISTICHIO DEL VERSO

a) finale verbale:

e no hai null amic qui negun gauig m'aport, 1,9

Ramon, si vós faits ço que a vós se cové, 9,1

no és culpa d'aquells de qui us anats clamant, 11,8

mortalment mantes vets de què em són confessat; 12,2

ab los molts grans senyors qui no us volon aidar, 13,5

Altri esempi: 9,10; 13,4 e 9; 24,11; 25,5 e 9; 33,10; 37,3; 38,9; 44,1; 48,2; 51,$12 ; 55,8 ; 59,12 ; 60,11 ; 61,3 ; 63,5 ; 68,3$ e $6 ; 69,1$.

Totale 25

b) finale nominale:

que hom fa envers vós, qui ens jutjats en la mort. 1,4

com a home irat que fuig a mal senyor, 4,4

e a teologia, la qual m'és mais coral; 8,6

Però parrà al judici qui haurà discreció, 16,11

Ramon li filosof qui foren antigament, 36,1

Altri esempi: 46,9; 47,9 e 11; 52,8; 56,7.

Totale 10

II. ESEMPI DI PROPOSIZIONI RELATIVE MINORI E MAGGIORI DI SEI SILLABE

a) verbali:

trisillabici: e si vós no havets null amic qui us ajut 6,6;20,5; 22,10;

quadrisillabici: que en siats despagat, car Cell qui tot ho ve, 9,5 e 6;20,6;

19 Per gli esempi occitano-catalani rimando a B. Spaggiari, La poesia religiosa anonima catalana o occitana, in Annali della scuola Normale Superiore di Pisa, serie III, 7, 1 (1977), pp. 117-350. 
pentasillabici: la fe sens paor de mort e qui ho sabran far. 24,12; 31,1; 37,3; 50,3 ;

ottosillabici: N'ermità, cell qui vol molt servir e honrar 34,2;

eneasillabici: l'ermità, qui el pregava con se degués jaquir 35,2 e 6; 26,5; 37,8; 40,11; 49,10; 56,8; 57,10;

decasillabici : e cells ab qui volets vostre fait acabar 19,7; 21,3;22,4; 40,10; 46,$4 ; 60,12$;

endecasillabici: per qui hom pot saber tota res natural 8,3; 45,5; 54,2 e 5;

dodecasillabici: que fas per honrar Déu e d'homens salvament. 7,12; 8,2; 19,$11 ; 25,6 ; 27,4 ; 29,7 ; 35,12 ; 44,5 ; 50,4 ; 55,4$.

Totale 39 esempi.

b) nominali:

trisillabici: per ço hai desplaer, car cells qui són major 32,8;

ottosillabici: car null home qui perdés tan preciós cabal, 8,11; 15,1;38,7;

eneasillabici: la honor que eu tracté per Déu tan llongament, 7,2 ; 15,2;

decasillabici: d'aquells a qui Déus ha donat mais d'honrament 7,7; 20,4; 63,3 ;

endecasillabici: e qui poden bastar a tuit nós e a llor, 32,7; 49,12;

dodecasillabici: que es presentà a mi cinc vets crucificat. 2,$4 ; 4,3 ; 6,3$ composto da 2 relative esasillabiche di cui la seconda dipendente; 9,12; 16,$12 ; 26,4 ; 32,6 ; 37,12 ; 56,2$ e $6 ; 59,9$. Totale 22 esempi.

Abbiamo così un totale di 96 esempi, pari al 11,6\% dei versi totali, una cifra molto inferiore a quella del Plant (23\%).

Passiamo ora ai casi di iperbato, forma usatissima da Llull sia nella poesia sia nella prosa.. Distinguiamo gli iperbati che si concludono nel singolo verso:

$\mathbf{1}, 5$ e $6 ; \mathbf{2}, 5 ; \mathbf{3}, 1,3,8$ e $10 ; \mathbf{4}, 12 ; \mathbf{5}, 2,3,4,5,8,9,11$ e $12 ; \mathbf{6}, 3 ; 7,2$ e $12 ; \mathbf{8}, 7$ e $9 ; \mathbf{9}, 11 ; \mathbf{1 0}, 1$ e $9 ; \mathbf{1 1}, 7,9,10,11$ e $12 ; \mathbf{1 2}, 3,5,6,9,10$ e 12 ; $\mathbf{1 3}, 2$ e $7 ; \mathbf{1 4}, 3,11$ e $12 ; \mathbf{1 5}, 11 ; \mathbf{1 6}, 2,3,4,9 ; \mathbf{1 7}, 1,4,6,11$ e $12 ; \mathbf{1 8}, 2,6$ e $7 ; \mathbf{1 9}, 1,2,5,7$ e $9 ; \mathbf{2 0}, 10 ; \mathbf{2 1}, 2,3$ e $10 ; \mathbf{2 2}, 2,5,6$ e $7 ; \mathbf{2 3}, 1,6,7,8$, e $9 ; \mathbf{2 4}, 2,4,7$ e $9 ; \mathbf{2 5}, 5$, e $9 ; \mathbf{2 6}, 1 ; \mathbf{2 7}, 2,4,5,9,11$ e $12 ; \mathbf{2 9}, 2,7,8$ e $9 ;$ $\mathbf{3 0}, 2,3,9$ e $12 ; \mathbf{3 1}, 8 ; \mathbf{3 2}, 6$ e $9 ; \mathbf{3 3}, 12 ; \mathbf{3 4}, 2,6,9$ e $11 ; \mathbf{3 5}, 7,10$ e 11 ; 36,2; 37, $1,3,7$ e $11 ; \mathbf{3 8}, 4 ; \mathbf{3 9}, 4,10$ e $12 ; \mathbf{4 0}, 10 ; \mathbf{4 1}, 2 ; \mathbf{4 2}, 1,2,7$ e 12 ; $\mathbf{4 5 , 3}$ e $9 ; \mathbf{4 6 , 3}, 8$ e $11 ; \mathbf{4 7 , 2 ,} 4$ e $12 ; \mathbf{4 8}, 2$ e $10 ; \mathbf{4 9}, 4$ e $10 ; \mathbf{5 0}, 5 ; \mathbf{5 1}, 5,9$, 10 e $11 ; \mathbf{5 2}, 2,3$ e $5 ; \mathbf{5 3}, 1 ; \mathbf{5 4}, 2,3,5,6,7$ e $10 ; \mathbf{5 5}, 4,6,7,9$ e $12 ; \mathbf{5 6}, 6$, 10 e $12 ; \mathbf{5 7}, 2$ e $11 ; \mathbf{5 8}, 2,3,4,10,11$ e $12 ; \mathbf{5 9}, 1,2,3,4 ; \mathbf{6 0}, 3,5,6,7,10$, 11,$12 ; \mathbf{6 1}, 1,2,3,4,5,8,9,10$ e $11 ; \mathbf{6 2}, 1,2,3,4,10,11$ e $12 ; \mathbf{6 3}, 12$; $\mathbf{6 4 , 5 , 8}$ e $9 ; \mathbf{6 5}, 7$ e $11 ; \mathbf{6 6}, 2,3,4,6,8,11$ e12; 67, 5, 8, 11e 12; 68, 1, 2, $3,4,7,11 ; \mathbf{6 9}, 1,6,7,8,9,10,11$; 
da quelli più complessi, che superano la misura del verso per esaurirsi nei successivi:

$\mathbf{3}, 11-12 ; \mathbf{5}, 6-7 ; \mathbf{7}, 4-6,9-10 ; \mathbf{8}, 5-6, \mathbf{1 0}, 6-7 ; \mathbf{1 1}, 4-5 ; \mathbf{1 3}, 4-6,8-9 ; \mathbf{1 4}, 5-$ $8 ; \mathbf{2 0}, 5-7 ; \mathbf{2 1}, 8-9 ; \mathbf{2 2}, 3-4 ; \mathbf{2 8}, 2-4 ; \mathbf{2 9}, 5-6 ; \mathbf{3 0}, 6-7$; 31,4-5; 33, 2-3; 35,4$5 ; \mathbf{3 6}, 4-5,10-11 ; \mathbf{3 7}, 8-9 ; \mathbf{3 9}, 8-9 ; \mathbf{4 0}, 6-7,8-9 ; \mathbf{4 1}, 10-12 ; \mathbf{4 3}, 7-8 ; \mathbf{4 4}, 3-4$; 47,6-7; 48,5.6, 8-9, 11-12; 52,7-8; 56,2-3, 4-5, 9-10; 57,9-10; 59,7-9; 69,2-3.

Da questa ampia serie si intuisce come l'iperbato sia la forma preferita da Llull per ottenere la rima, nel Desconhort ancor più che nel Plant, visto che la maggior parte degli esempi qui riportati sono frutto dello spostamento del verbo a fine verso, quindi in posizione di rima. Sono quindi confermati nuovamente $i$ dati raccolti da Rubió i Balaguer ${ }^{20}$ sulla prosa rimata lulliana e viene convalidata anche l'affermazione di Ruffini che Llull non si sia interessato a ottenere rime verbali o nominali, ma a piegare i concetti che voleva esprimere nella rigida, e a volte monotona, forma dell'alessandrino, riuscendo a dar loro movimento e respiro poetico.

$$
* \quad * \quad *
$$

Vediamo ora di trarre delle conclusioni, anche in questo caso provvisorie visto che l'analisi si potrà concludere solo con lo spoglio completo delle opere rimate del beato majorchino. Nel Desconhort si dimostra ancora l'amore di Llull per la facilità: le rime sono 20 su 69 strofe, di cui 13 comuni con il Plant. Non si riscontrano rime difficili, anche se Ruffini considera «rima non facile $»^{21}$ -or e -ors che sono usate in sei strofe. Per facilitare il confronto tra i due testi prendo anch'io come esempio questa rima. Escluse le parole-rima: deshonor, plor, vós, llausor, preicador (-ors), menor (-ors), secor (-ors), error (-ors) che si ripetono due volte; tristor, amor, valor (-ors), dolor, llor tre volte; honor, senyor (-ors) quattro volte; pecador (-ors) che si ripete cinque volte e considerando che la str. 41 in -or presenta l'unico caso verbale, otteniamo un totale di 43 rimanti su 72; inoltre l'assonanza tra -os e -ors è marcata, nel Desconhort, da dodici rimanti su un totale di 23 contro l'unico caso del Plant; il che pone l'accento sull'abilità di Llull nel destreggiarsi anche con rime non facili e parole-rima. L'analisi comparativa dei due poemi ha fatto emergere altri dati interessanti. Llull, infatti, riutilizza 92 rimanti del Plant nel Desconhort, distribuendo le rime del primo nel secondo - cfr. in particolare str. 2, 9, 20, 32 del Plant con str. 6, 57, 67, 14 del Desconhort-, inoltre i mezzi usati per ottenere la rima sono gli stessi nelle due poesie e, ancora una volta, si dimostra la destrezza poetica del beato nel trovare rime omofone, contrari e sinonimi in rima. La differenza principale tra i due poemi la troviamo nella quantità di rime verbali rispetto a quelle nominali. In effetti, il Desconhort mostra una predilezione per le rime verbali che sono il mezzo principalmente usato dal beato per ottenere la rima nelle prose rimate. Ancora non si possono trarre conclusioni certe, ma sicuramente si può affermare che Llull non ebbe un interesse nel trovare rime

\footnotetext{
${ }^{20}$ J. Rubió i Balaguer, cit., p. 243.

${ }^{21}$ M. Ruffini, Osservazioni, cit. p. 22
} 
nominali o verbali per costruire le sue poesie, ma che cercò un metodo efficace per rendere piacevoli e facilmente memorizzabili i suoi poemi. Quest'ultimo aspetto lo interessava particolarmente, infatti lo stesso Desconhort è: «en rimes posat, per ço que no s'oblit» ${ }^{22}$. La poesia era uno strumento efficacissimo di diffusione del suo messaggio e permetteva un accesso più facile allo studio dell'Arte, ne sono un esempio le Regles introductòries a la pratica de l'Art dimostrativa citate supra. Certo Llull non desiderava che le sue poesie fossero cantate dai trovatori profani che disprezzava fortemente, quindi si fece fautore di una nuova figura: il Juglar de Valor $^{23}$, che canterà le lodi di Dio e, naturalmente, le poesie di Llull. Per confermare l'importanza che il beato dava all'Arte memorativa - che Llull aveva intenzione di compilare, ma che non realizzò mai-, possiamo leggere il capitolo De duratione nella terza parte della Rhetorica Nova:

«Duplex est verborum duratio. Una in vocis actualitate consistens, que tanta est quanta fuerit continua verborum prolatio. Alia vero est secundum quod in hominum memoria retinentur qui, audita verba retinentes in memoria et intelligencia cognoscentes ad ea affecti, ipsa post modum recitant. Unde, si ad finem debitum — ut decet— fuerint recitata, erunt verba pulcra, quemadmodum dicta evangelica et omnes Sacre Scripture et gesta gloriosorum virorum qui, etsi a presenti seculo migraverint, vivunt memoria.. ${ }^{24}$.

Recenti studi di filologia cognitiva sul rapporto tra rima e memoria ${ }^{25}$ hanno dimostrato che l'uso della rima non è solo un vezzo estetico ma uno strumento fondamentale per aiutare l'attività mnemonica, come è espresso anche nelle Leys d'amor: «entendem pauzar alqus yssemples e alcunas difinitios per maniera de rims per so qu'om los puesca plus leu reportar e decorar. ${ }^{26}$ Questi fenomeni sono pienamente riflessi nel testo che stiamo analizzando e sono un interessantissimo campo di ricerca nell'opera di Llull.

Dall'analisi sintattica si è notato che l'ampio uso della proposizione relativa dimostra la necessità di spiegare delle teorie complesse o di chiarire dei temi che non erano stati capiti. I molti casi di iperbato sembrerebbero allontanarci dall'amore per il trobar leu, ma anche in questo caso, come nel Plant, sono resi necessari dal bisogno di rinchiudere un testo - e quindi un pensierocomplesso nel rigido schema delle strofe in alessandrini e di adattare il tutto a

22 str. $69,3$.

23 Il contrastato rapporto con i trovatori è manifestato in vari passi dell'opera lulliana, cfr. cap. 118 del Llibre de contemplació; il Blaquerna, dove compare il Juglar de Valor; la str. 61 del Desconhort; ecc.

${ }^{24}$ R. Llull, Ramon Llull's New Rhetoric: Text and Translation of Llull's Rethorica Nova, a cura di M. D. Johnston. Hermagoras Press, Davis (California) 1994, p. 35.

25 P. Canettieri, Metrica e memoria, in Rivista di filologia cognitiva (2005) in http://w3.uniroma1.it/cogfil/metrica.html; e G. Santini, Rima e memoria, in Rivista di filologia cognitiva (2005) in http://w3.uniroma1.it/cogfil/rima.html.

26 M. Gatien-Arnoult, Monuments de la Littérature Romane. Les flors del Gay Saber, estier dichas Las Leys d'Amors, 3 vols., París-Tolosa 1841-1843, vol. I, p. 3. 
una melodia musicale, oltre al bisogno di dare ritmo alle strofe. Il Plant e il Desconhort mostrano quindi delle affinità non solo superficiali, ma sono collegati da legami ben più forti:

«En les dues obres existia total adequació entre el sentiment del poeta i la forma emprada per a expressar-lo. Per això se'n val. No pas per un desig infeliç d'imitació de maneres literàries a la moda. Ramon Llull el que fa és emular-les, segur en la seva fe que les podrà superar per la virtut de la seva inspiració religiosa.. ${ }^{27}$

Queste affinità si riscontrano anche nel titolo dato al Plant nel ms. Ottoboniano Latino 845 del XIV secolo, conservato nella biblioteca apostolica vaticana: «De la passió de Iesuchrist e lo desconort que ac nostra dona de son fil» $[\mathrm{f} .43 \mathrm{r}]$.

Infine, l'interesse per le forme provenzali mi ha indotto ad allargare l'indagine di Ruffini con l'analisi dei provenzalismi in rima. Come sappiamo, all'epoca di Llull i copisti erano già avvezzi a conservare le tinte occitaniche nelle poesie, in particolare se la modifica di queste comportava irregolarità metriche o di rima. Nel Desconhort troviamo dunque trenta parole occitane in rima, senza contare le cinque che corrisponderebbero a casi di declinazione bicasuale e altre che sono equivalenti nelle due lingue. La cifra non è molto alta in confronto al Plant (dove i provenzalismi sono 23 e i casi di declinazione 16), sembra più che il beato usi queste forme per mancanza di sinonimi nella propria lingua o per necessità di rima. Questo tipo di analisi richiede un'indagine approfondita in tutti i mss. e sarà ripresa in altra sede.

Sperando di non aver trasformato il grande Sconforto autobiografico del Dottore Illuminato in uno sterile elenco di numeri, mi auguro di aver fatto luce su alcuni aspetti che, in un'indagine più ampia, si potrebbero rivelare utilissimi per capire come si creava poesia nel medioevo.

\section{RIMARIO}

$\begin{array}{lll} & \text { NominaLe } & \text { VerbaLE } \\ \text {-al } & \text { General } 8,1 & \text { val } 8,5 \text { e } 7 \\ & \text { espirital } 8,2 & \text { cal } 8,9 \\ & \text { natural } 8,3 \text { e } 8 & \\ & \text { sensual } 8,4 & \\ \text { coral } 8,6 & \\ \text { mortal } 8,10 & \\ \text { cabal } 8,11 & \\ \text { terrenal } 8,12 & \end{array}$

27 J. Rubió i Balaguer, Estudis literaris, Edicions 62, Barcelona 1996, p. 109. 


$\begin{array}{cl}\text {-ança } & \text { esperança } 46,1 \\ & \text { balança } 46,2 \\ & \text { malanança } 46,3 \\ & \text { pietança } 46,4 \\ & \text { bonança } 46,5 \\ & \text { alegrança } 46,6 \\ & \text { fiança 46,7 e } 11 \\ & \text { començança } 46,8 \\ & \text { semblança } 46,9 \\ & \text { tristança } 46,10 \\ & \text { amistança } 46,12\end{array}$

-an,-ant semblant 11,1

talant 11,$2 ; 67,9$

benestant 11,5

enpecant 11,6

tant 11,7

avant 11,9

quant 11,10

semblant 11,12

afan 67,1

an 67,2

gran 67,3

dan 67,7
-ar car 19,3
pecar 19,10
clar 24,9
amar 34,4
estar 39,3
joglar 59,7; 61,7
rimar 59,9

dubtant 11,3

malestant 11,4

clamant 11,8

començant 11,$11 ; 67,12$

genollant 67,4

plorant 67,5

deman 67,6

coman 67,8

mostrant 67,10

preïcant 67,11 procurar 13,$1 ; 17,6 ; 59,3 ; 65,5$

membrar 13,2; 39,6; 65,1

dubtar 13,3; 59,10

acabar 13,$4 ; 17,2$ e $12 ; 19,7 ; 34,6 ; 59,2$

aidar 13,5

amar 13,$6 ; 19,1 ; 24,6 ; 39,8 ; 61,5 ; 65,10$

menar 13,$7 ; 19,9 ; 34,7$

rancurar 13,8

encolpar 13,9; 19,12; 24,2;61,10

desconhortar 13,10

esforçar 13,11

honrar 13,12; 17,4; 19,11; 34,1; 39,9;

61,$4 ; 65,2$ 
far 17,$1 ; 23,4$ e $12 ; 24,12 ; 34,9 ; 59,4$;

61,$8 ; 65,11$

donar 17,3

clamar 17,$5 ; 24,4$

estar 17,$7 ; 34,10 ; 59,5 ; 61,12 ; 65,7$

pensar 17,8

preicar 17,$9 ; 23,9 ; 24,11 ; 27,1$

consellar 17,$10 ; 34,5$

anar 17,$11 ; 27,6$

parlar 19,$2 ; 27,7 ; 39,4$

nomenar 19,4

llausar 19,5

menysprear 19,$6 ; 39,10 ; 59,11 ; 61,6$

escoltar 19,8; 59,6

aconhortar 23,1

plorar 23,2

utjar 23,3

par 23,5

provar 23,6; 24,1

trobar 23,7; 39,2; 59,12

marturiar 23,8

maravellar 23,10

atorgar 23,11

mostrar 24,3

argumentar 24,5

Encarnar 24,7

contrastar 24,8

recobrar 24,10

batejar 27,2

ordenar 27,3

escapar 27,4

disputar 27,5

enterpretar 27,8

enançar 27,9

trigar 27,10

pregar 27,11

contemplar 27,12

lleixar 34,2 


$$
\begin{aligned}
& \text { enutjar 34,3 } \\
& \text { començar } 34,8 \\
& \text { guasanyar } 34,11 \\
& \text { entristar } 34,12 \\
& \text { consolar } 39,1 \text { e } 5 \\
& \text { bastar } 39,7 \\
& \text { alegrar } 39,11 \\
& \text { confortar } 39,12 \\
& \text { tornar } 61,1 \\
& \text { aportar } 61,2 \\
& \text { portar } 61,3 \\
& \text { retornar } 61,9 \\
& \text { escusar } 61,11 \\
& \text { gloriejar } 65,3 \\
& \text { crear } 65,4 \\
& \text { benestar } 65,6 \\
& \text { despertar } 65,9 \\
& \text { venjar } 65,12 \\
& \text { amenar } 59,1 \\
& \text { cantar 59,8 } \\
& \text { abissar } 65,8
\end{aligned}
$$

-às $\quad \operatorname{las} 22,5$

cas 22,12 celàs 22,1

provàs 22,2

alvàs 22,3

amàs 22,4

estudiàs 22,6

oblidàs 22,7

passàs 22,8

fas 22,9

membràs 22,10

dubtàs 22,11

enamorat 2,6

-at vanitat 2,$1 ; 12,8$

pecat 2,$2 ; 12,6$ e $10 ; 30,8$;

44,9

preïcat 2,7

carnalitat 2,3

encarnat 2,9

pietat 2,$4 ; 33,9 ; 57,4 ; 68,4$ honrat 2,$11 ; 26,3 ; 51,11 ; 55,5$ 
crucificat 2,5

veritat 2,$8 ; 26,2 ; 30,1$;

44,$12 ; 57,1$ e 8

volentat 2,$10 ; 26,12 ; 33,4$

grat 2,$12 ; 18,7 ; 45,7 ; 51,3$;

66,10

cobeitat 18,1

paupertat 18,5

ciutat 18,8

regnat 18,$10 ; 63,10$

altetat 26,4

creat 26,8

increat 26,9

bontat 26,$11 ; 38,7$

llibertat 30,$3 ; 45,3,5$ e 11

potestat 30,5

caritat 30,$6 ; 66,5$

errat 30,10

fat 33,8

estat 38,3

necessitat 38,5; 45,8

deitat 38,10

mirvat 38,12

contrarietat 44,4

possibilitat 44,5

autrejat 44,11

lletrat 45,$1 ; 55,3$

predestinat 45,2 e 12

heretat 51,2

prelat 55,$10 ; 63,4$

tractat 57,3

crestiantat 63,3 e $9 ; 68,12$

quantitat 63,7

sarraïnitat 63,8

comiat 66,1

tempestat 68,1

societat 68,10 pecat 12,1

confessat 12,2

revelat 12,3

contat 12,4

confermat 12,5

ajutat 12,$9 ; 30,11$

amat 12,$11 ; 30,2 ; 45,9 ; 55,1,68,7$

desemparat 12,12

habitat 18,2

llarguejat 18,4

reptat 18,6

excusat 18,9

acabat 18,$11 ; 44,6 ; 63,2$

escoltat 18,12

creat 26,$1 ; 38,4$ e $11,45,4$

gloriat 26,5

provat 26,6

membrat 26,$7 ; 55,2 ; 66,4$

dat 26,$10 ; 68,3$

forçat 30,$4 ; 45,6$

pagat 30,$7 ; 38,6$

lligat 30,9

desconsolat 30,12; 57,6

anat 33,$1 ; 57,9$

enançat 33,$2 ; 68,11$

meravellat 33,3

fadigat 33,5

assajat 33,6

assenyat 33,7

ujat 33,10

reposat 33,11

consolat 33,$12 ; 38,1 ; 44,8 ; 51,1$

irat 38,2

mirvat 38,8

trobat 38,9

predestinat 44,1 


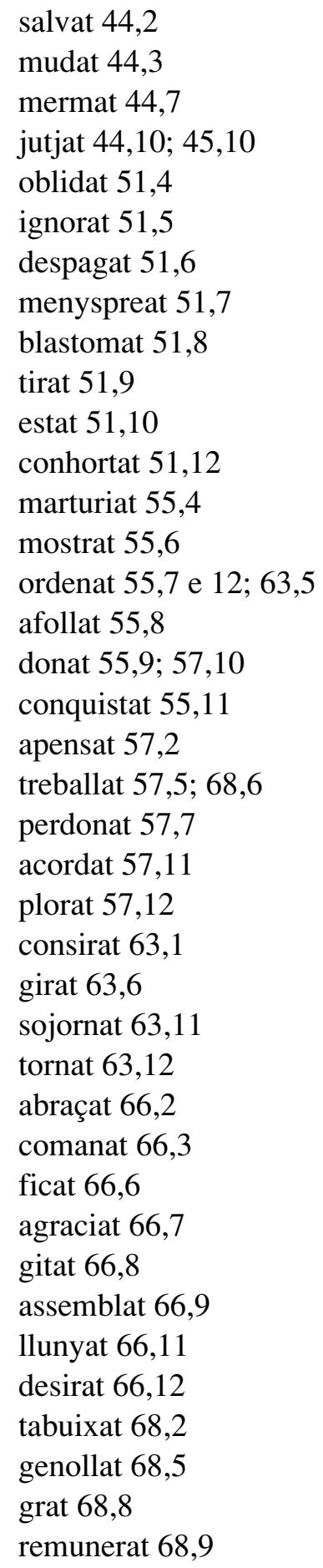




$\begin{array}{lll}\text {-ats } & \text { malvats } 42,4 & \text { estiats } 42,1 \\ \text { llats } 42,5 & \text { hajats } 42,2 \\ \text { pietats } 42,6 & \text { aujats } 42,3 \\ \text { caritats } 42,8 \text { e } 11 & \text { siats } 42,7 ; 54,3 \\ \text { damnats } 42,10 & \text { salvats } 42,9 \\ \text { desirats } 54,1 & \text { consolats } 42,12 \\ & \text { honrats } 54,2 \\ & \text { procurats } 54,4 \\ & \text { menats } 54,5 \\ & \text { treballats } 54,6 \text { e } 8 \\ & \text { viviats } 54,7 \\ & \text { desviats } 54,9 \\ & \text { digats } 54,10 \\ & \text { estats } 54,11 \\ & \text { amats } 54,12 \\ & \\ \text { bé } 9,2 \text { e } 11 ; 25,3 & \text { cové } 9,1 \text { e } 4 ; 25,2 \\ \text { dessé } 9,6 ; 25,4 & \text { ve (venir) } 9,3 ; 25,5 \\ \text { se } 9,8 & \text { ve (vedere) } 9,5 ; 25,6 \\ \text { mercé } 9,9 & \text { capté } 9,7 \\ \text { fe } 9,12 ; 25,1 \text { e } 7 & \text { reté } 9,10 \\ \text { re } 25,11 & \text { conté } 25,8 \\ & \text { manté } 25,9 \\ & \text { té } 25,10 \\ & \text { descové } 25,12\end{array}$

-ent estament 3,$1 ; 15,2 ; 28,1$;

36,$11 ; 47,1$

descreent 3,$2 ; 40,2$

concebiment 3,2

eixament 3,4

ordenament 3,$5 ; 43,8$;

47,$3 ; 53,8 ; 56,1$

preïcament 3,6 ;

argument 3,$7 ; 28,2$ e 7 ;

56,9

exalçament 3,8

convertiment 3,$9 ; 7,4 ; 28$

verament 3,10 dolent 3,$11 ; 53,5$

entenent 15,4

reprendent 15,6

convinent 15,11

coneixent 36,2

ment 36,6

vivent 36,$10 ; 50,6$

desobeent 40,7

consent 43,9

present 47,4 
languiment 3,12; 43,4

compliment 7,$1 ; 15,1$;

28,$12 ; 36,9 ; 50,11$

perdent 50,3

llongament 7,2; 28,9

clamament 7,3

Moniment 7,5; 56,11

defalliment 7,6

honrament 7,7; 43,12;

50,$12 ; 53,3$

nient 7,$8 ; 28,3 ; 36,8$;

50,$5 ; 53,12$

follament 7,9; 47,11

enteniment 7,$10 ; 36,5$

procurament 7,11

salvament 7,$12 ; 53,10$

discretament 15,3

rancurament 15,$5 ; 40,11$

saviament 15,7

exauçament 15,8

breument 15,$9 ; 47,9$

falliment 15,$10 ; 36,7 ; 40,10$

pacient 15,$12 ; 50,1$

honestament 28,4

disputament 28,6; 56,5

gent 28,$8 ; 53,9 ; 56,3$

mantinent 28,10; 47,5

Espirament 28,11

antigament 36,1

profitament 36,3

començament 36,4; 53,6

següent 36,12

justament 40,1

desolament 40,3

jutjament 40,$4 ; 43,6$

malament 40,$5 ; 56,8$

falsament 40,6

amament 40,8

turment 40,9; 43,2; 50,4

dretament 40,12

jausent 53,4 
parlament 43,1

oblitament 43,3

advocament 43,5

egalment 43,7

minvament 43,10

espavent 43,11

avilament 47,2

marriment 47,$6 ; 53,7$

consolament 47,$7 ; 50,2 ; 53,11$

clarament 47,8

gabament 47,10

desesperament 47,12

sanament 50,7

vestiment 50,8

pagament 50,9

impacient 50,10

principalment 53,1

gloriejament 53,2

enantiment 56,2

uniment 56,4 e 10

tractament 56,6 e 12

vivent 56,7

-er

poder 52,$1 ; 58,4 ; 64,12$

esper 52,6

noncaler 52,$7 ; 64,3$

desplaer 52,8

captener 52,9

voler 52,11

despler 58,6

saber 58,8

ver 64,6

parer 64,9

doler 64,10

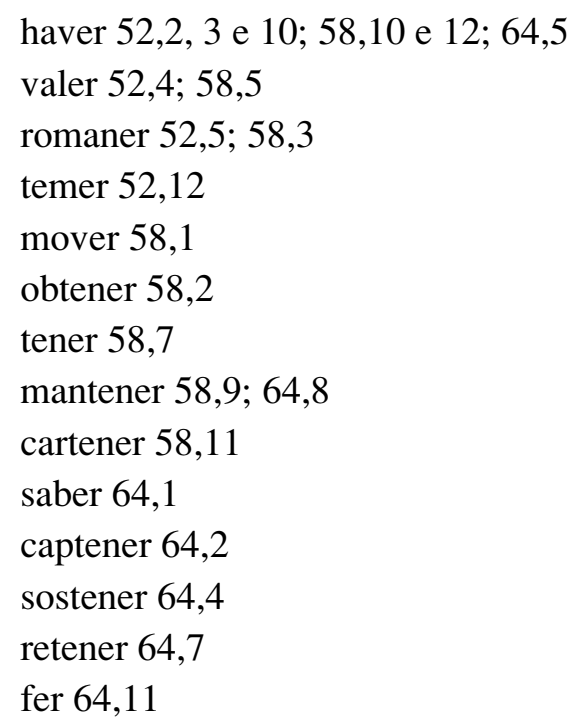




\begin{tabular}{|c|c|c|}
\hline -es & $\begin{array}{l}\text { tres } 62,4 \\
\text { res } 62,5 \\
\text { marquès } 62,7\end{array}$ & $\begin{array}{l}\text { reprès } 62,1 \\
\text { estés } 62,2 \\
\text { escomès } 62,3 \text { e } 9 \\
\text { tengués } 62,6 \\
\text { pogués } 62,8 \\
\text { estegués } 62,10 \\
\text { fes } 62,11 \\
\text { presés } 62,12\end{array}$ \\
\hline -ets & & $\begin{array}{l}\text { irets } 60,1 \\
\text { procurarets } 60,2 \\
\text { havets } 60,3,10 \text { e } 11 \\
\text { serets } 60,4 \\
\text { volets } 60,5 \\
\text { valets } 60,6 \\
\text { dubtets } 60,7 \\
\text { fèts } 60,8 \\
\text { devets } 60,9 \\
\text { perdets } 60,12\end{array}$ \\
\hline -ia & $\begin{array}{l}\text { malencolia } 5,1 \\
\text { fellonia } 5,11 \\
\text { Maria } 48,3 \\
\text { jerarquia } 48,4 \\
\text { dia } 48,5 \\
\text { via } 48,7 \text { e } 9 \\
\text { pia } 48,10\end{array}$ & $\begin{array}{l}\text { venia } 5,2 \text { e } 7 \\
\text { havia } 5,3 \text { e } 6 \\
\text { valia } 5,4 \\
\text { paria } 5,5 \\
\text { podia } 5,8 \\
\text { sentia } 5,9 \\
\text { consolaria } 5,10 \\
\text { poria } 5,12 ; 48,1 \\
\text { planyia } 48,2 \\
\text { sia } 48,6 \text { e } 11 \\
\text { deuria } 48,8 \\
\text { estia } 48,12\end{array}$ \\
\hline -ir & $\begin{array}{l}\text { desir } 31,12 \\
\text { martir } 35,4 \\
\text { albir } 35,9\end{array}$ & $\begin{array}{l}\text { retenir } 31,1 \\
\text { convertit } 31,2 \\
\text { ausir } 31,3 ; 35,1 \text { e } 10 \\
\text { dir } 31,4 ; 35,3 \text { e } 11 \\
\text { servir } 31,5 ; 35,5 \\
\text { seguir } 31,6 ; 35,7\end{array}$ \\
\hline
\end{tabular}


fallir 31,7

destruir 31,8

devenir 31,9

partir 31,10

jaquir 31,$11 ; 35,2$

romanir 35,6

fenir 35,8

enantir 35,12

-it $\quad$ Esperit 29,2; 69,12

escrit 29,4

convertit 29,1

ardit 69,4

ausit 29,3

sentit 29,5

unit 29,6

departit 29,7; 69,9

establit 29,8; 69,7

consentit 29,9;69,8

fallit 29,10

exausit 29,11

aunit 29,12

escrit 69,1

dit 69,2

oblit 69,3

complit 69,5

requerit 69,6

abellit 69,10

contradit 69,11

-ó $\quad$ discreció 16,1 e 11

raó 16,2 e $8 ; 20,8 ; 37,9$

só 16,4 e 9; 20,7; 37,11

fallió 16,3

perdó 20,9

companyó 16,5

fo 20,$10 ; 37,1$ e 3

pro 16,$6 ; 20,6$

bandó 16,7

sermó 16,10; 20,11

perdó 16,12 ;

entenció 20,1

estimació 20,2

presumpció 20,3

opinió 20,$4 ; 37,2$ e 6 
bo 20,5 e $12 ; 37,4$

Encarnació 37,5

unió 37,7

producció 37,8

visió 37,10

resurreccó 37,12

-or tristor 4,$1 ; 41,5 ; 49,12 \quad$ aor 41,2

deshonor 4,$2 ; 49,5$

amor 4,$3 ; 41,4 ; 49,1$

senyor 4,$4 ; 41,1 ; 49,3$

plor 4,$5 ; 41,8$

dolor 4,6; 41,6; 49,6

dolçor 4,7

clamor 4,8

pecador 4,$9 ; 41,10 ; 49,2$ e 7

honor 4,$10 ; 32,6 ; 41,3 ; 49,4$

fervor 4,11

valor 4,$12 ; 32,5$

preïcador 32,1

menor 32,2

prior 32,3

mellor 32,4

llor 32,$7 ; 41,11 ; 49,8$

major 32,8

llausor 32,9; 49,10

cor 32,10

follor 32,11

Creador 32,12

secor 41,7

consolador 41,9

perdonador 41,12

error 49,9

austor 49,10

-ors, -os consirós 10,1

joiós 10,2

valors 10,3 
amadors 10,4

amargors 10,5

ajudadors 10,6

errors 10,7

cabalós 10,8

vós 10,$9 ; 14,8$

virtuós 10,10

viciós 10,11

secors 10,12

ociós 14,1

pecadors 14,2

possessiós 14,3

llangors 14,4

messiós 14,5

Preïcadors 14,6

Menors 14,7

senyors 14,9

dubtós 14,10

pererós 14,11

piadós 14,12

-ort desconhort 1,1 e 7

conhort 1,2

tort 1,3

m'aport 1,9

mort 1,4

port 1,10

fort 1,5

port 1,6

deport 1,8

sort 1,11

confort 1,12

-ut salut 6,$2 ; 21,10$

perdut 6,$1 ; 21,11$

vertut 6,$4 ; 21,9$

vengut 6,3

abatut 6,5

ajut 6,6 e 12; 21,7

haüt 6,7

decebut 6,$8 ; 21,2$

acorregut 6,9

vençut 6,10 


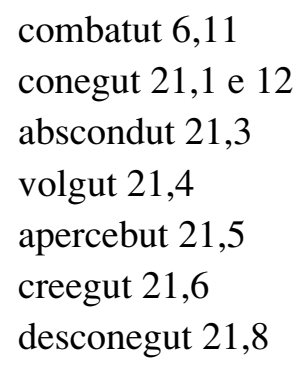

\section{BIBLIOGRAFIA}

Abràmova, M. A., Lo desconhort de Ramon Llull en el context de la literatura hagiogràfica i autobiogràfica medieval, in Actes de l'Onzè Co loqui Internacional de Llengua i Literatura Catalanes, Palma (Mallorca), Publicacions de l'Abadia de Montserrat, 1998, I, pp. 93-97.

Antonelli R., Equivocatio e repetitio nella lirica trobadorica, in Id., Seminario romanzo, Bulzoni, Roma 1979.

ANTONELli R., Rima equivoca e tradizione rimica nella poesia di Giacomo da Lentini. I. Le canzoni, in Bollettino del Centro di Studi Filologici e Linguistici Siciliani, 13 (1977) pp. 20-106.

Bonner A. e Badia L., Ramón Llull. Vida, pensamiento y obra literaria, Sirmio Quaderns Crema, Barcellona 1993 [Trad. aggiornata e corretta di Id., Ramon Llull. Vida, pensament i obra literària, Les naus d'Empúries, Palma de Mallorca Barcelona 1988].

Bonner A., Obres selectes de Ramon Llull (1232-1316), 2 vols, Moll, Palma de Mallorca, 1989.

CANETTIERI P., Metrica e memoria, in Rivista di filologia cognitiva (2005), disponibile in http://w3.uniroma1.it/cogfil/metrica.html

Gatien-Arnoult M., Monuments de la Littérature Romane. Les flors del Gay Saber, estier dichas Las Leys d'Amors, 3 vols., París-Tolosa 1841-1843.

LLull R., Libre de Evast e Blanquerna, a cura di S. Galmés, ENC, Barcino, Barcelona, (I) 1935; (II) 1947; (III-IV) 1954.

Llull R., Lo desconhort, Cant de Ramon, a cura di J. Batalla, Obrador Edèndum, Barcelona 2004.

Llull R., Lo sconforto, a cura di M. Ruffini, Il Melograno, Fussi Sansoni, Firenze 1953.

LLuLl R., Poesies, a cura di R. d'Alòs-Moner, ENC, Barcino, Barcelona 1928.

LLull R., Ramon Llull's New Rhetoric: Text and Translation of Llull's Rethorica Nova, a cura di M. D. Johnston. Hermagoras Press, Davis (California) 1994.

MeneGhetTi M. L., Il pubblico dei trovatori, Einaudi, Torino 1992. 
PAgÈs A., Le «Desconort» ou le «Découragement» de Ramon Llull, in Annales du Midi, 50 (1938) pp. 113-156, 225-267.

PARRAMON I BlASCO J., Repertori mètric de la poesia catalana medieval, Curial Edicions Catalanes, Publicacions de l'Abadia de Montserrat, Barcelona 1992.

Rubió I BALAguer J., Estudis literaris, Edicions 62, Barcelona 1996.

Rubió i Balaguer J., Ramon Llull i el lul.lisme, Publicacions de l'Abadia de Montserrat 1985.

Rubió i Balaguer J., Sobre la poesia rimada de Ramon Llull, in Estudios dedicados a Menéndez Pidal V, Madrid 1954, pp. 307-318 [anche in id., Ramon Llull i el lul.lisme, Publicacions de l'Abadia de Montserrat 1985, pp. 234-247].

RUfFINI M., Osservazioni sulla rima finale del «Plant de Nostra Dona Santa Maria», in EL, 10 (1966), pp.129-140; 11 (1967) pp.21-30.

SANTINI G., Rima e memoria, in Rivista di filologia cognitiva (2005), disponibile in http://w3.uniroma1.it/cogfil/rima.html

Spaggiari B., La poesia religiosa anonima catalana o occitana, in Annali della scuola Normale Superiore di Pisa, serie III, 7, 1 (1977), pp.117-350. 